\title{
Proposition of determination of displacements using the TDRA 6000 laser station
}

\author{
Jacek Sztubecki ${ }^{1, *}$, Maria Mrówczyńska ${ }^{2}$, and Adam Bujarkiewicz ${ }^{1}$ \\ ${ }^{1}$ University of Science and Technology, Faculty of Civil and Environmental Engineering and Architecture, 7 prof. S. Kaliski St., \\ 85-796 Bydgoszcz, Poland \\ ${ }^{2}$ University of Zielona Góra, Institute of Building Engineering, Department of Land and Environment Engineering, 9 Licealna St., \\ 65-417 Zielona Góra, Poland
}

\begin{abstract}
With modern measurement technologies, it is possible to achieve higher precision and solve complex measurement tasks. The tested device (TDRA 6000 laser station) features a direct drive technology ensuring a 3D point accuracy of $0.25 \mathrm{~mm}$. TDRA 6000 combined with proper software is a perfect tool to monitor engineering objects. The article presents the measurement technique and the method of calculation of 3D displacements of control and measurement networks using the TDRA 6000 laser station from Leica. The values presented herein derive from periodic measurements carried out in control points stabilised at a ground the above-ground crossing between buildings located on the premises of the University of Science and Technology campus in Bydgoszcz.
\end{abstract}

\section{Introduction}

Geodetic monitoring with measurements and their interpretation makes it possible to specify conclusions on the dynamics of changes in objects $[1,2]$. The basic symptom of adverse phenomena occurring on buildings includes vertical displacements of control and measurement network points [3]. Contemporary measurement methods can be applied to complete tasks which could not be fulfilled with the traditional methods, while ensuring the required accuracy. Furthermore, measurements conducted with such techniques are less labour intensive and less time consuming [4].

Displacements and deformations of engineering structures can be determined by using modern laser devices. They allow to get better accuracy and give the ability to solve complex measurement tasks. Today, these technologies are often used in metrological industrial measurements [4]. They are characterized by high precision, reaching $+/-15 \mu \mathrm{m}+6 \mu \mathrm{m} / \mathrm{m}$ when measuring with a retroreflector and operating range up to $120 \mathrm{~m}$ [5]. These parameters are obtained for measurements of average size of structures.

The article presents an analysis of results of vertical and horizontal displacement measurements carried out with the TDRA 6000 laser station from Leica. The station features a direct drive technology ensuring a 3D point accuracy of $0.25 \mathrm{~mm}$. The producer's inspection certificate in accordance with DIN 55350-18-4.2.2 specifies the following accuracy for this device: maximum deviation of distance measurement of $+/-0.2$ $\mathrm{mm}$ (standard deviation of single distance measurement $\sigma=0.1 \mathrm{~mm})$ and standard deviation of angle measurement (Hz and $\mathrm{V}$ ) $\sigma=0.15$ mgon [6]. The device combined with proper software is a perfect tool to monitor engineering objects.

Methods applied in measurements may constitute an alternative solution to measurements conducted with classic methods. The article also presents the way of stabilisation of measurement points. With an analysis of measurement data, it was possible to make conclusions on the measurement technique and precision.

\section{Tested objects}

Displacement determination measurements with the use of the TDRA 6000 laser station were conducted on two control networks established on the buildings of the University of Science and Technology in Bydgoszcz.

The existing benchmarks stabilised on the external walls of the 3.1 building were adapted to the first network (Fig. 1).

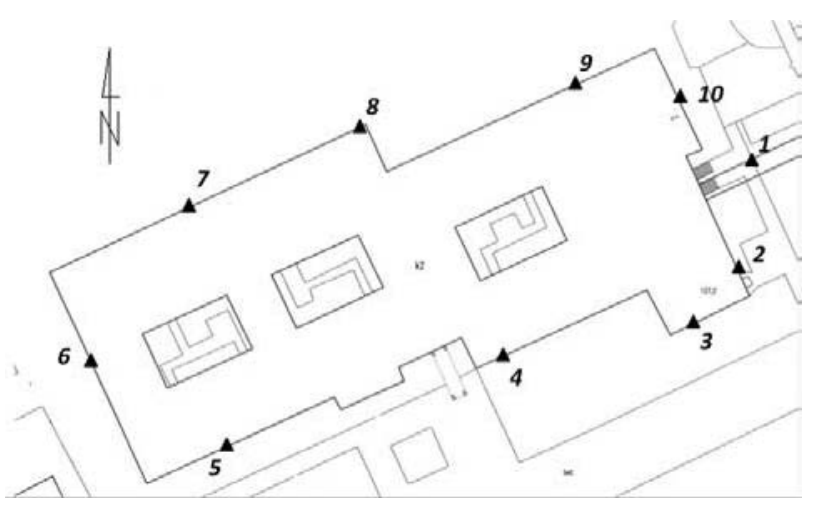

Fig. 1. Benchmarks arranged on the 3.1 building.

\footnotetext{
* Corresponding author: jaceksz@utp.edu.pl
} 
Steel nuts used to stabilise a 1.5 " red-ring-reflector were permanently fixed on the benchmarks (Photo 1).
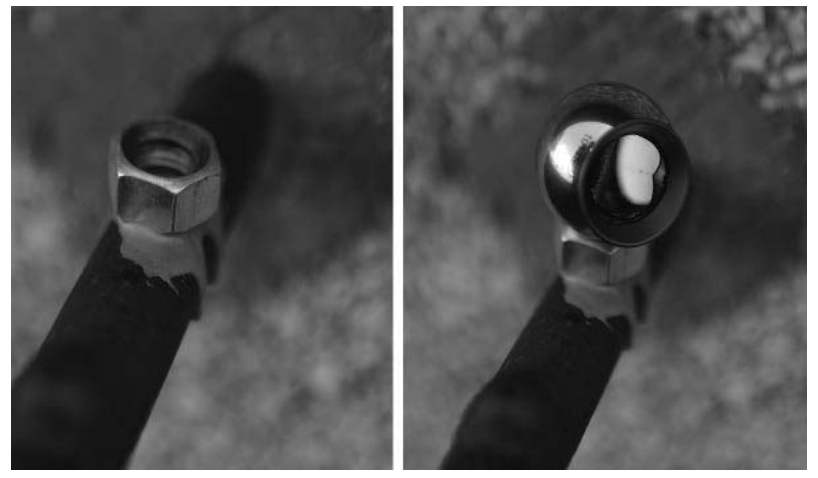

Photo. 1. 1.5" red-ring-reflector stabilisation.

In order to determine vertical displacements, the difference in elevation between the respective points was measured. The measurements were conducted at equal target distances not exceeding $30 \mathrm{~m}$. Each measurement on every stand was carried out at least two times, while changing the laser station height and making sure that the deviation from elevation differences was permissible.

The second network was established inside aboveground crossing between buildings which connects the Auditorium Novum building with the 2.1 building (Photo 2).

Above-ground crossing between buildings is of a reinforced concrete design supported on 12 columns. The network points were stabilised with washers (Photo 3 ) fixed to the floor of above-ground crossing and the adjacent buildings. The measurement network arrangement layout is shown in Fig. 2.

The measurements were conducted on 7 free stands (Fig. 2) while observing the position of the 1.5" red-ringreflector and recording $3 \mathrm{D}$ coordinates of the control points. Each measurement stand was used twice, moving slightly the measuring device.

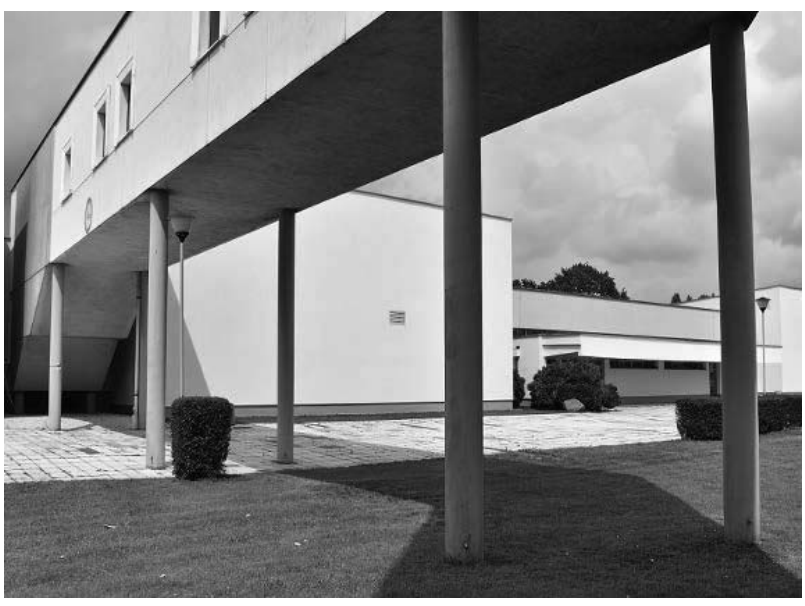

Photo. 2. The above-ground crossing between buildings.

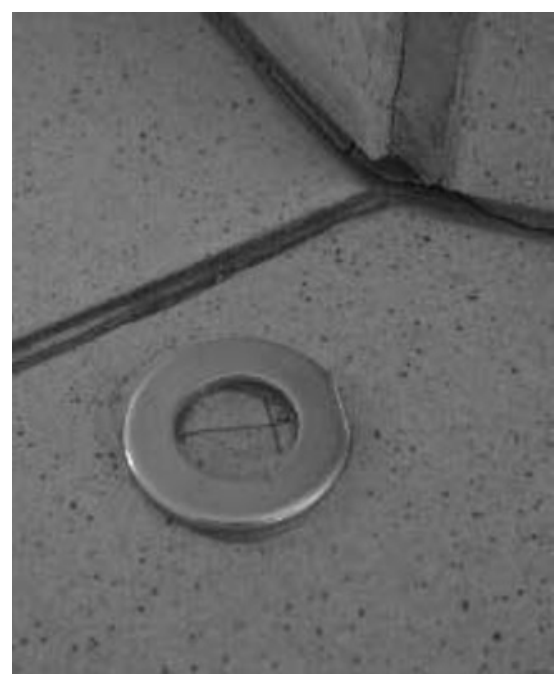

Photo. 3. Stabilisation of the control points on the floor inside the above-ground crossing between buildings.

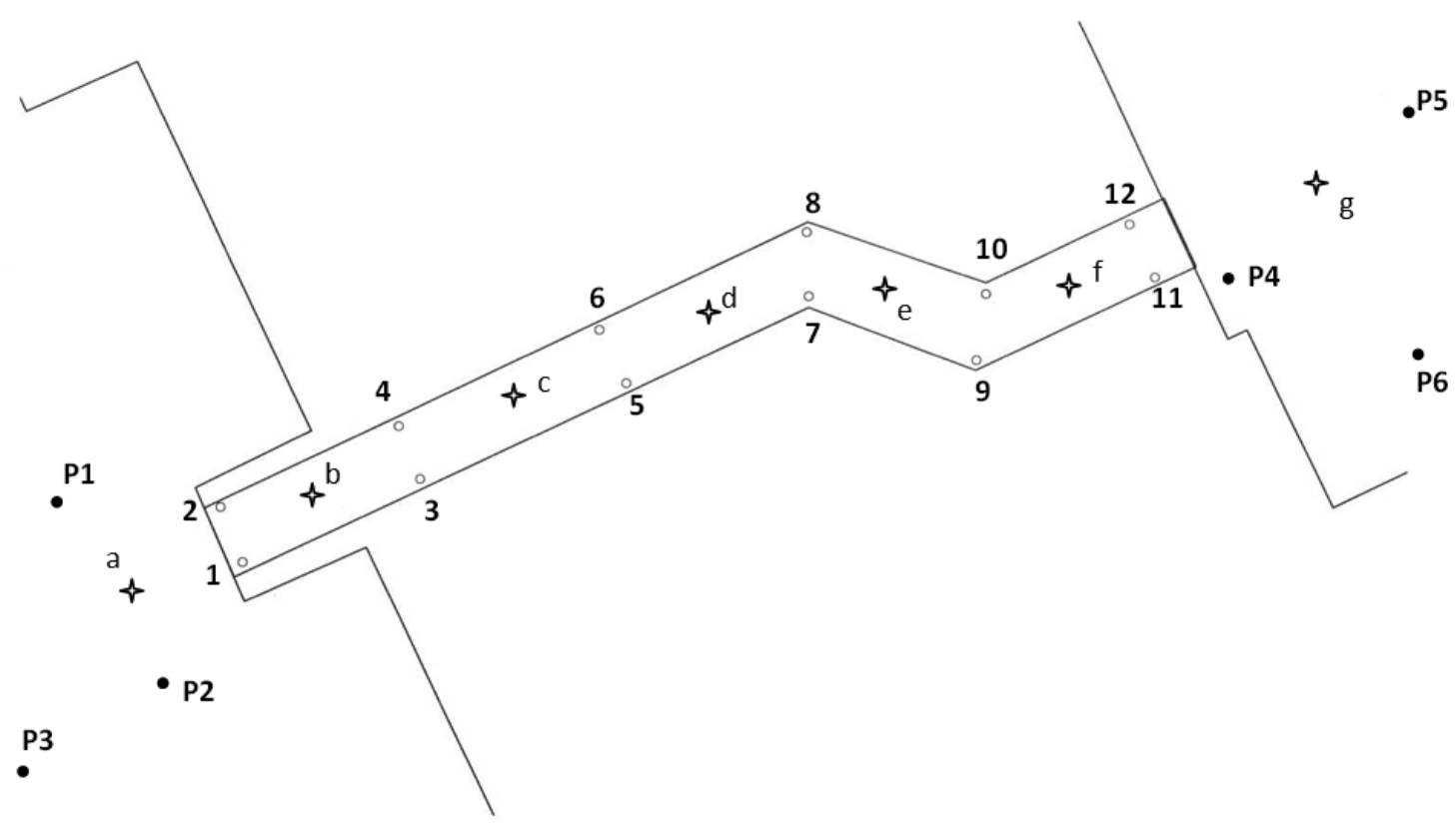

Fig. 2. Control point arrangement layout $(\mathrm{P} 1 \div \mathrm{P} 6$ - reference points, $1 \div 12-$ control points, $\mathrm{a} \div \mathrm{g}-$ instrument stand). 


\section{Test results}

Four measurement series were conducted for the first network. An initial measurement check was performed following each series by summarising and adding up elevation differences in a circuit. The differences in the height and value concerning not-closing of the mesh of the measured network was presented in Table 1.

Due to the lack of points showing stability in the height range in the vicinity of the examined object, in the process of leveling the network the relative vertical displacements were measured.

Relative displacement values and their errors are presented in Table 2. It can be noticed that errors of determined displacements do not exceed $0.20 \mathrm{~mm}$, which is within the generally accepted limit of vertical displacement measurement accuracy.

In the adjustment process, the error values of measured height differences were also determined. The maximum values of these errors were:

- 1 measurement -2 measurement $-0.03 \mathrm{~mm}$,

- 1 measurement -3 measurement $-0.08 \mathrm{~mm}$,

- 1 measurement -4 measurement $-0.06 \mathrm{~mm}$.

The procedure for the second network involved two measurements of $3 \mathrm{D}$ point coordinates on each of 7 stands (a-g). Only the nearest control points were observed from each station: for the outermost stands (a, g) -5 points, for the remaining stands -4 points.

Two measurement series were conducted for which calculations of spatial control point displacements were made at two stages.

At the first stage, differences in elevation between the network points were calculated and then subjected to rigorous adjustment. Results obtained at this stage and vertical displacement errors are shown in Table 3.

Table. 1. Results of network measurement checks for individual measurement series.

\begin{tabular}{|c|c|c|c|c|c|}
\hline \multicolumn{6}{|c|}{ Elevation difference [mm] } \\
\hline \multirow{2}{*}{ Point } & \multicolumn{4}{|c|}{ measurements } & \multirow{2}{*}{ Point } \\
\hline & 1 & 2 & 3 & 4 & \\
\hline \multirow{2}{*}{1} & & & & & 1 \\
\hline & -18.0 & -18.1 & -18.3 & -18.3 & \multirow{2}{*}{2} \\
\hline 2 & 377.2 & 377.2 & 377.0 & 376.8 & \\
\hline 3 & \multirow{2}{*}{1223.6} & \multirow{2}{*}{1224.2} & \multirow{2}{*}{1224.0} & \multirow{2}{*}{1223.7} & 3 \\
\hline 4 & & & & & 4 \\
\hline \multirow{2}{*}{5} & 5.1 & 5.1 & 5.0 & 3.9 & 5 \\
\hline & -295.7 & -295.7 & -295.5 & -295.4 & \multirow{2}{*}{6} \\
\hline 6 & \multirow{2}{*}{253.0} & 253.0 & 253.5 & 253.6 & \\
\hline 7 & & & & & 7 \\
\hline 8 & -178.2 & -178.3 & -178.4 & -178.3 & 8 \\
\hline 9 & 62.8 & 62.8 & 63.2 & 63.8 & 9 \\
\hline & -321.2 & -321.2 & -321.2 & -321.0 & \\
\hline 10 & - & - & - & - & 10 \\
\hline 1 & 1108.6 & 1109.1 & 1108.9 & 1108.5 & 1 \\
\hline Total & 0.0 & -0.1 & 0.4 & 0.3 & \\
\hline
\end{tabular}

Table. 2. Values of displacements and error of displacements.

\begin{tabular}{|c|c|c|c|c|c|c|c|}
\hline \multirow{3}{*}{ Point } & \multicolumn{6}{|c|}{ Displacements and error of displacements [mm] } & \multirow{3}{*}{ Point } \\
\hline & \multicolumn{2}{|c|}{$\begin{array}{l}1 \text { measurement - } \\
2 \text { measurement }\end{array}$} & \multicolumn{2}{|c|}{$\begin{array}{c}1 \text { measurement - } \\
3 \text { measurement }\end{array}$} & \multicolumn{2}{|c|}{$\begin{array}{l}1 \text { measurement - } \\
4 \text { measurement }\end{array}$} & \\
\hline & dZ & mdZ & dZ & $\mathbf{m d Z}$ & dZ & $\mathbf{m d Z}$ & \\
\hline 1 & 0.08 & 0.04 & 0.58 & 0.16 & 0.76 & 0.16 & 1 \\
\hline 2 & -0.01 & 0.03 & 0.24 & 0.12 & 0.43 & 0.12 & 2 \\
\hline 4 & 0.61 & 0.03 & 0.36 & 0.12 & 0.07 & 0.12 & 4 \\
\hline 5 & 0.62 & 0.04 & 0.22 & 0.16 & -1.16 & 0.16 & 5 \\
\hline 6 & 0.63 & 0.05 & 0.38 & 0.18 & -0.89 & 0.18 & 6 \\
\hline 7 & 0.64 & 0.05 & 0.84 & 0.20 & -0.32 & 0.20 & 7 \\
\hline 8 & 0.55 & 0.04 & 0.60 & 0.18 & -0.45 & 0.18 & 8 \\
\hline 9 & 0.56 & 0.05 & 0.96 & 0.20 & 0.52 & 0.20 & 9 \\
\hline 10 & 0.57 & 0.05 & 0.92 & 0.18 & 0.69 & 0.18 & 10 \\
\hline
\end{tabular}


Table. 3. Results of network measurement checks for individual measurement series.

\begin{tabular}{|c|c|c|}
\hline Point & $\begin{array}{c}\mathbf{d Z} \\
{[\mathbf{m m}]}\end{array}$ & $\begin{array}{c}\mathbf{m d Z} \\
{[\mathbf{m m}]}\end{array}$ \\
\hline 1 & 0.15 & 0.07 \\
\hline 2 & 0.08 & 0.07 \\
\hline 3 & -0.05 & 0.07 \\
\hline 4 & -0.09 & 0.07 \\
\hline 5 & -0.13 & 0.08 \\
\hline 6 & -0.22 & 0.08 \\
\hline 7 & -0.01 & 0.08 \\
\hline 8 & 0.17 & 0.08 \\
\hline 9 & -0.07 & 0.07 \\
\hline 10 & 0.06 & 0.07 \\
\hline 11 & 0.03 & 0.07 \\
\hline 12 & -0.18 & 0.07 \\
\hline
\end{tabular}

The second stage involved determination of horizontal displacements of the established network. Calculations were made with flat X, Y coordinates of the control points in the following sequence:

1. an isometric transformation of points obtained from two positions of the laser station (separately for each measurement station) was performed for each free stand;

2. an isometric transformation combining (integrating) the free stands into one unit was performed for each measurement series;

3. a Helmert transformation was performed on 6 adjustment points for both measurement series - the purpose of the transformation was to present control point coordinates in a uniform system of coordinates;

4. a transformation of control point coordinates was performed for both measurement series up to the system of an axis parallel to the section connecting points 1 and 7;

5. $d X$ and $d Y$ displacements were calculated between the measurement series as differences of $\mathrm{X}, \mathrm{Y}$ coordinates.

As a result of the calculations, horizontal displacements of the control points were obtained. These are presented in Table 4.

An analysis of the accuracy of determined displacements was based on a transformation error. The value of this error was defined for points 1 and 3 of the calculation order.

Errors were determined for isometric transformation calculations carried out in accordance with point 1 . The value of these errors is presented in Table 5.

An error of $0.49 \mathrm{~mm}$ was determined for Helmert transformation calculations carried out in accordance with point 3 .
Table. 4. Results of horizontal displacements.

\begin{tabular}{|c|c|c|}
\hline Point & $\begin{array}{c}\mathbf{d X} \\
{[\mathbf{m m}]}\end{array}$ & $\begin{array}{c}\mathbf{d Y} \\
{[\mathbf{m m}]}\end{array}$ \\
\hline 1 & 0.45 & 0.94 \\
\hline 2 & 0.44 & 0.85 \\
\hline 3 & 0.16 & 1.18 \\
\hline 4 & 0.22 & 1.13 \\
\hline 5 & -0.35 & 0.77 \\
\hline 6 & -0.35 & 0.66 \\
\hline 7 & -0.87 & 0.19 \\
\hline 8 & -0.84 & 0.11 \\
\hline 9 & -1.57 & 0.19 \\
\hline 10 & -1.69 & 0.14 \\
\hline 11 & -1.89 & 0.28 \\
\hline 12 & -2.09 & 0.13 \\
\hline
\end{tabular}

Table. 5. Results of horizontal displacements.

\begin{tabular}{|c|c|c|}
\hline \multirow{2}{*}{ Stand } & \multicolumn{2}{|c|}{ Transformation error [mm] } \\
\cline { 2 - 3 } & $\begin{array}{c}\text { 0 measurement } \\
\mathbf{2 0 1 7 . 0 7 . 2 4}\end{array}$ & $\begin{array}{c}\text { 1 measurement } \\
\mathbf{2 0 1 7 . 0 9 . 0 9}\end{array}$ \\
\hline a & 0.11 & 0.08 \\
\hline b & 0.23 & 0.16 \\
\hline c & 0.20 & 0.21 \\
\hline d & 0.10 & 0.24 \\
\hline e & 0.15 & 0.08 \\
\hline f & 0.12 & 0.05 \\
\hline g & 0.28 & 0.19 \\
\hline
\end{tabular}

\section{Summary and conclusions}

The article presents possible applications of the TDRA 6000 laser station from Leica designed to determine vertical and horizontal displacements of engineering buildings.

Vertical displacement measurements covered a network of benchmarks located outside the 3.1 building which formed a closed circuit. The results confirmed the expected measurement accuracy and indicated that an average elevation difference error did not exceed 0.13 $\mathrm{mm}$ already at the network control stage when checking the circuit closure. Further analysis involved determination of observation errors and uncertainties based on rigorous adjustment of the measured network. It indicated that average errors of measured elevation differences did not exceed $0.12 \mathrm{~mm}$ and the error of determined displacements was not greater than $0.20 \mathrm{~mm}$.

Analysed measurement results of vertical displacements of the control points located inside the above-ground crossing between buildings show their high accuracy of $0.08 \mathrm{~mm}$. Horizontal displacements of this network were determined with isometric and Helmert transformations. Both isometric transformation 
errors for the free stands ( $\max 0.28 \mathrm{~mm}$ ) and the Helmert transformation error between the measurement series $(0.49 \mathrm{~mm})$ indicate high accuracy of the applied measurement method.

Measurement accuracy achieved when carrying out the test confirms that the employed methods constitute an alternative to classic displacement determination methods.

\section{References}

1. T. Lazzarini, Geodetic measurements of deformations and their application in construction, (PPWK, Warsaw, 1961, in Polish)

2. B. Wolski, Metrological monitoring of geotechnical objects (Publishing House of Krakow University of Technology, Krakow, 2006, in Polish)

3. R. Kadaj, Models, methods and algorithms for kinematic networks in geodetic measurements of displacements and deformation of objects (Publishing House of Krakow University, Krakow, 1998, in Polish)

4. A. Bujarkiewicz., J. Sztubecki, M. Sztubecka, Materiały Budowlane 503(7), 50-51, 2014

5. G.J. Wojcik, S.A. Lakanen, Proceedings of the 7th International Workshops on Accelerator Alignment, SPring-8, Japan, 2002

6. Leica Geosystems, http://metrology.leicageosystems.com/, access10 July, 2017 